\title{
Synthesis of certain pyrrole derivatives as antimicro-bial agents
}

\author{
MOSAAD SAYED MOHAMED ${ }^{1}$ \\ RAMDAN AHMED EL-DOMANY2 \\ RANIA HELMY ABD EL-HAMEED*1 \\ 1 Organic Chemistry Department \\ Faculty of Pharmacy, Helwan University \\ Cairo, Egypt \\ ${ }^{2}$ Microbiology and Immunology \\ Department, Faculty of Pharmacy \\ Helwan University, Cairo, Egypt
}

In an effort to establish new pyrroles and pyrrolo[2,3- $d]$ pyrimidines with improved antimicrobial activity we report here the synthesis and in vitro microbiological evaluation of a series of pyrrole derivatives. A series of new 2-aminopyrrole-3-carbonitriles (1a-d) were synthesized from the reaction of benzoin, primary aromatic amines and malononitrile, from which a number of pyrrole derivatives (2a-d to $5 \mathbf{a}-\mathbf{d}$ ) and pyrrolo[2,3-d]pyrimidines (6a-d to $10 \mathrm{a}, \mathrm{d}$ ) were synthesized. The in vitro antimicrobial testing of the synthesized compounds was carried out against Gram-positive, Gram-negative bacteria and fungi. Some of the prepared compounds, [2-amino-1-(2-methylphenyl)-4,5-diphenyl-1H-pyrrole-3-carbonitriles (1b), 2-amino-3-carbamoyl-1-(3-methylphenyl)-4,5-diphenyl-1H-pyrroles (2b), N-(3-cyano-1-(2-methylphenyl)-4,5-diphenyl-1H-pyrrol-2-yl)-acetamides (3b), N-(3-cyano-1-(3-methylphenyl)-4,5-diphenyl-1H-pyrrol-2-yl)-acetamides (3c), 2-amino-1-(4-methoxyphenyl)-4,5-diphenyl-3-tetrazolo-1H-pyrroles (5d), 7-(4-methoxyphenyl)-5,6-diphenyl-7H-pyrrolo [2,3-d]pyrimidin-4(3H)-ones (7d), 7-(3-methylphenyl)-5,6-diphenyl-7H-pyrrolo[2,3-d]pyrimidin-4(3H)-thione $(9 \mathbf{b})$ and $N$-(7-(2-methylphenyl)-5,6-diphenyl-7H-pyrrolo[2,3-d] pyrimidine)- $N$-aryl amines (10a)] showed potent antimicrobial activity.

Keywords: pyrrole, pyrrolopyrimidine, antimicrobial activity

For several decades, interest in pyrrole derivatives as antimicrobial agents has led to the preparation and antimicrobial evaluation of hundreds of such molecules. For example, monodeoxypyoluteorin and 2-(2'-hydroxybenzoyl) pyrrole bromine derivatives are pyrrole derivatives having antimicrobial activity against Staphylococcus aureus, Bacillus subtilis and Escherichia coli and an interesting antifungal activity against Candida albicans, as it was shown by Petruso et al. (1) and Raimondi et al. (2). 3,4,5,3,5-Pentabromo-

\footnotetext{
* Correspondence; e-mail: zeiadomar@yahoo.com
} 
-2-(2-hydroxybenzoyl)pyrrole, a synthetic antibacterial compound related to pyrrolomycins, has significant activity against Staphylococcus epidermidis and Staphylococcus aureus, as proved by D. Schillaci et al. (3).

2-Methyl-1,3,5-trisubstituted pyrroles have significant activity against Mycobacterium tuberculosis, as proved by Biava et al. $(4,5)$. Diguanidino-1-methyl-2,5-diaryl-1H-pyrrole derivatives have antifungal activity against Candida species, as reported by Jana et al. (6).

Tubercidin, toyocamycin and sangivamycin are naturally occurring pyrrolo[2,3-d]pyrimidine antibiotics having significant activity against Mycobacterium tuberculosis, Candida albicans and Streptococcus neoformans, which was shown in many reports (7-9).

2,4-Diamino-5-methyl-6-substituted-pyrrolo[2,3- $d$ ]pyrimidines are potent and selective dihydrofolate reductase (DHFR) inhibitors against Pneumocystis carinii, Toxoplasma gondii and Mycobacterium avium, as reported by Gangjee et al. (10).

The aim of this study was to synthesize new pyrrole and pyrrolo[2,3-d] pyrimidine derivatives, hoping that they could be of promising chemical and biological interest.

\section{EXPERIMENTAL}

All melting points are uncorrected and were measured using an Electro-thermal IA 9100 apparatus (Shimadzu, Japan). IR spectra were recorded as potassium bromide pellets on a Perkin-Elmer 1650 spectrophotometer (USA), ${ }^{1} \mathrm{H}$ NMR spectra were determined on a Varian Mercury (300 MHz) spectrometer (Varian, UK) and chemical shifts were expressed as ppm against TMS as internal reference. Mass spectra were recorded on a 70 eV EI Ms-QP 1000 EX (Shimadzu). Microanalyses were operated using Vario, Elementar apparatus (Shimadzu) and the results were within accepted range $( \pm 0.40 \%)$ of the calculated values.

Physicochemical and spectral data for the synthesized compounds are given in Tables I and II. The target compounds were synthesized as outlined in Schemes 1 and 2. Synthesis of compound 1 was performed according to literature $(11,12)$.

\section{Synthesis of 2-amino-4,5-diphenyl-1-substituted-1H-pyrrole-3-carbonitriles (1a-d)}

A mixture of benzoin $(2 \mathrm{~g}, 0.01 \mathrm{~mol})$, the appropriate amine [aniline $(0.93 \mathrm{~g}, 0.01 \mathrm{~mol})$, $o$-toluidine or $m$-toulidine $(1.17 \mathrm{~g}, 0.01 \mathrm{~mol})$, or $p$-anisidine $(1.23 \mathrm{~g}, 0.01 \mathrm{~mol})]$ and conc. $\mathrm{HCl}(6-8$ drops) in toluene $(50 \mathrm{~mL})$ was heated under reflux for $6 \mathrm{~h}$ and cooled. Malononitrile $(0.66 \mathrm{mg}, 0.01 \mathrm{~mol})$ was added, followed by a catalytic amount $(1.5 \mathrm{~mL})$ of pyridine portionwise and left to reflux until a solid was formed. The solvent was evaporated under reduced pressure and the residue was recrystallized from methanol to give compounds 1a-d, respectively.

\section{Synthesis of 2-amino-1-aryl-3-carbamoyl-4,5-diphenyl-1H-pyrroles (2a-d)}

An appropriate cyanopyrrole, 1a (3.35 g, $0.01 \mathrm{~mol}), \mathbf{1 b}, \mathbf{1 c}(3.49 \mathrm{~g}, 0.01 \mathrm{~mol})$ or $\mathbf{1 d}$ (3.65 $\mathrm{g}, 0.01 \mathrm{~mol})$ in conc. sulphuric acid $(40 \mathrm{~mL})$, was refluxed for $4 \mathrm{~h}$, cooled, poured 
onto ice-water, neutralized with ammonia to give compounds $2 \mathrm{a}-\mathrm{d}$, respectively in the form of precipitates which were filtered, dried, and recrystallized from methanol.

\section{Synthesis of N-(1-aryl-3-cyano-4,5-diphenyl-1H-pyrrol-2-yl)-acetamides (3a-d)}

The appropriate aminopyrrole, $1 \mathrm{a}(3.35 \mathrm{~g}, 0.01 \mathrm{~mol}), \mathbf{1 b}, \mathbf{1 c}(3.49 \mathrm{~g}, 0.01 \mathrm{~mol})$ or $\mathbf{1 d}$ $(3.65 \mathrm{~g}, 0.01 \mathrm{~mol})$, in acetic anhydride $(40 \mathrm{~mL})$ was refluxed for $4 \mathrm{~h}$, cooled, poured onto ice-water, neutralized with ammonia to give compounds 3a-d, respectively in the form of precipitates which were filtered off, dried, and recrystallized from methanol.

\section{Synthesis of 3-(2-amino-1-aryl-4,5-diphenyl-1H-pyrrole)amidoximes $(4 c, d)$}

The appropriate cyanopyrrole 1c $(3.49 \mathrm{~g}, 0.01 \mathrm{~mol})$ or $1 \mathrm{~d}(3.65 \mathrm{~g}, 0.01 \mathrm{~mol})$, hydroxyl amine hydrochloride $(0.33 \mathrm{~g}, 0.01 \mathrm{~mol})$ and anhydrous sodium carbonate $(5.3 \mathrm{~g}, 0.05 \mathrm{~mol})$ in absolute ethanol $(40 \mathrm{~mL})$ was refluxed for $4 \mathrm{~h}$, filtered while hot and the residue was washed with hot ethanol. The collected filtrate was cooled, poured onto ice-water to yield precipitates, which were filtered, dried, and recrystallized from methanol, to give compounds $4 \mathrm{c}, \mathrm{d}$, respectively.

\section{Synthesis of 2-amino-1-aryl-4,5-diphenyl-3-tetrazolo-1H-pyrroles $(5 a, b, d)$}

A mixture of the appropriate cyanopyrrole 1a (3.35 g, $0.01 \mathrm{~mol}), \mathbf{1 b}(3.49 \mathrm{~g}, 0.01 \mathrm{~mol})$ or $1 \mathrm{~d}(3.65 \mathrm{~g}, 0.01 \mathrm{~mol})$, sodium azide $(0.65 \mathrm{~g}, 0.01 \mathrm{~mol})$ and ammonium chloride $(1.06 \mathrm{~g}$, $0.02 \mathrm{~mol}$ ) was refluxed in DMF $(30 \mathrm{~mL})$ for $4 \mathrm{~h}$, filtered while hot and the residue was washed with hot DMF. The collected filtrate was concentrated, cooled, poured onto ice-water to yield precipitates, which were filtered, dried and recrystallized from methanol, to give compounds $\mathbf{5 a}, \mathbf{b}, \mathbf{d}$, respectively.

\section{Synthesis of 5,6-diphenyl-7-substituted-7H-pyrrolo[2,3-d]pyrimidin-4-yl-amines (6a-d)}

A mixture of the appropriate aminopyrrole 1a $(3.35 \mathrm{~g}, 0.01 \mathrm{~mol}), \mathbf{1 b}, \mathbf{1 c}(3.49 \mathrm{~g}, 0.01$ $\mathrm{mol})$ or $1 \mathrm{~d}(3.65 \mathrm{~g}, 0.01 \mathrm{~mol})$ and formamide $(30 \mathrm{~mL}, 0.066 \mathrm{~mol})$ was heated under reflux for $6 \mathrm{~h}$, cooled and poured onto ice-water to give precipitates, which were filtered off, dried, and recrystallized from ethanol to yield compounds $6 a-d$, respectively.

\section{Synthesis of 5,6-diphenyl-7-substituted-7H-pyrrolo[2,3-d]pyrimidin-4(3H)-ones (7a-d)}

An appropriate aminopyrrole, 1a $(3.35 \mathrm{~g}, 0.01 \mathrm{~mol}), \mathbf{1 b}, \mathbf{1 c}(3.49 \mathrm{~g}, 0.01 \mathrm{~mol})$ or $\mathbf{1 d}$ $(3.65 \mathrm{~g}, 0.01 \mathrm{~mol})$, in formic acid $(20 \mathrm{~mL}, 85 \%)$ was heated under reflux for $3 \mathrm{~h}$, cooled, poured onto ice-water to give compounds $7 \mathrm{a}-\mathrm{d}$ in the form of precipitates which were filtered off, dried, and recrystallized from ethanol.

\section{Synthesis of 4-chloro-5,6-diphenyl-7-substituted-7H-pyrrolo[2,3-d]pyrimidine (8a-d)}

An appropriate pyrrolopyrimidinone, 7a (3.63 g, $0.01 \mathrm{~mol}), 7 \mathbf{b}, 7 \mathrm{c}(3.77 \mathrm{~g}, 0.01 \mathrm{~mol})$ or $7 \mathbf{d}(3.93 \mathrm{~g}, 0.01 \mathrm{~mol})$, was heated under reflux in phosphorus oxychloride $(30 \mathrm{~mL})$ for 
M. S. Mohamed et al:: Synthesis of certain pyrrole derivatives as antimicrobial agents, Acta Pharm. 59 (2009) 145-158.

Table I. Elemental analysis of the newly prepared compounds 1-10

\begin{tabular}{|c|c|c|c|c|c|c|c|c|c|c|}
\hline \multirow{2}{*}{$\begin{array}{l}\text { Compd. } \\
\text { No. }\end{array}$} & \multirow{2}{*}{$\mathrm{R}$} & \multirow{2}{*}{$\mathrm{R}^{\prime}$} & \multirow{2}{*}{$\begin{array}{l}\text { Yield } \\
(\%)\end{array}$} & \multirow{2}{*}{$\begin{array}{l}\text { M.p. } \\
\left({ }^{\circ} \mathrm{C}\right)\end{array}$} & \multirow{2}{*}{$\begin{array}{l}\text { Mol. formula } \\
\qquad\left(M_{\mathrm{r}}\right)\end{array}$} & \multicolumn{5}{|c|}{ Microanalysis calcd./found (\%) } \\
\hline & & & & & & $\mathrm{C}$ & $\mathrm{H}$ & $\mathrm{N}$ & $\mathrm{Cl}$ & $\mathrm{S}$ \\
\hline $1 \mathrm{a}$ & $\mathrm{H}$ & - & 86 & $248-250(13)$ & $\begin{array}{c}\mathrm{C}_{23} \mathrm{H}_{17} \mathrm{~N}_{3} \\
(335.41)\end{array}$ & - & - & - & - & - \\
\hline $1 b$ & $2-\mathrm{CH}_{3}$ & - & 39 & $210-212$ & $\begin{array}{c}\mathrm{C}_{24} \mathrm{H}_{19} \mathrm{~N}_{3} \\
(349.44)\end{array}$ & $\begin{array}{l}82.49 \\
82.83\end{array}$ & $\begin{array}{l}5.48 \\
5.28\end{array}$ & $\begin{array}{l}12.03 \\
12.22\end{array}$ & $\begin{array}{l}- \\
-\end{array}$ & - \\
\hline 1c & $3-\mathrm{CH}_{3}$ & - & 58 & 197-199 & $\begin{array}{c}\mathrm{C}_{24} \mathrm{H}_{19} \mathrm{~N}_{3} \\
(349.44)\end{array}$ & $\begin{array}{l}82.49 \\
82.74\end{array}$ & $\begin{array}{l}5.48 \\
5.62\end{array}$ & $\begin{array}{l}12.03 \\
11.98\end{array}$ & $\begin{array}{l}- \\
-\end{array}$ & - \\
\hline $1 d$ & $4-\mathrm{OCH}_{3}$ & - & 91 & 204-206 & $\begin{array}{c}\mathrm{C}_{24} \mathrm{H}_{19} \mathrm{~N}_{3} \mathrm{O} \\
(365.44)\end{array}$ & $\begin{array}{l}78.89 \\
78.58\end{array}$ & $\begin{array}{l}5.24 \\
5.39\end{array}$ & $\begin{array}{l}11.50 \\
11.22\end{array}$ & $\begin{array}{l}- \\
-\end{array}$ & - \\
\hline $2 a$ & $\mathrm{H}$ & - & 72 & 207-209 & $\begin{array}{c}\mathrm{C}_{23} \mathrm{H}_{19} \mathrm{~N}_{3} \mathrm{O} \\
(353.43)\end{array}$ & $\begin{array}{l}78.16 \\
78.50\end{array}$ & $\begin{array}{l}5.42 \\
5.12\end{array}$ & $\begin{array}{l}11.89 \\
12.23\end{array}$ & - & - \\
\hline $2 b$ & $2-\mathrm{CH}_{3}$ & - & 44 & $231-233$ & $\begin{array}{c}\mathrm{C}_{24} \mathrm{H}_{21} \mathrm{~N}_{3} \mathrm{O} \\
(367.45)\end{array}$ & $\begin{array}{l}78.46 \\
78.21\end{array}$ & $\begin{array}{l}5.76 \\
5.63\end{array}$ & $\begin{array}{l}11.44 \\
11.82\end{array}$ & - & - \\
\hline $2 c$ & $3-\mathrm{CH}_{3}$ & - & 59 & 196-198 & $\begin{array}{c}\mathrm{C}_{24} \mathrm{H}_{21} \mathrm{~N}_{3} \mathrm{O} \\
(367.45)\end{array}$ & $\begin{array}{l}78.45 \\
78.15\end{array}$ & $\begin{array}{l}5.76 \\
5.43\end{array}$ & $\begin{array}{l}11.44 \\
11.61\end{array}$ & - & - \\
\hline $2 d$ & $4-\mathrm{OCH}_{3}$ & - & 79 & $229-231$ & $\begin{array}{c}\mathrm{C}_{24} \mathrm{H}_{21} \mathrm{~N}_{3} \mathrm{O}_{2} \\
(383.45)\end{array}$ & $\begin{array}{l}75.18 \\
75.39\end{array}$ & $\begin{array}{l}5.52 \\
5.22\end{array}$ & $\begin{array}{l}10.96 \\
10.56\end{array}$ & - & - \\
\hline $3 a$ & $\mathrm{H}$ & - & 81 & $234-236$ & $\begin{array}{c}\mathrm{C}_{25} \mathrm{H}_{19} \mathrm{~N}_{3} \mathrm{O} \\
(377.45)\end{array}$ & $\begin{array}{l}86.20 \\
86.54\end{array}$ & $\begin{array}{l}5.43 \\
5.08\end{array}$ & $\begin{array}{l}8.38 \\
8.64\end{array}$ & - & - \\
\hline $3 b$ & $2-\mathrm{CH}_{3}$ & - & 55 & 193-194 & $\begin{array}{c}\mathrm{C}_{26} \mathrm{H}_{21} \mathrm{~N}_{3} \mathrm{O} \\
(391.48)\end{array}$ & $\begin{array}{l}86.18 \\
85.89\end{array}$ & $\begin{array}{l}5.79 \\
5.34\end{array}$ & $\begin{array}{l}8.04 \\
8.29\end{array}$ & - & - \\
\hline $3 c$ & $3-\mathrm{CH}_{3}$ & - & 67 & 203-205 & $\begin{array}{c}\mathrm{C}_{26} \mathrm{H}_{21} \mathrm{~N}_{3} \mathrm{O} \\
(391.48)\end{array}$ & $\begin{array}{l}86.18 \\
86.31\end{array}$ & $\begin{array}{l}5.79 \\
5.46\end{array}$ & $\begin{array}{l}8.04 \\
8.36\end{array}$ & - & - \\
\hline $3 d$ & $4-\mathrm{OCH}_{3}$ & - & 87 & 184-186 & $\begin{array}{c}\mathrm{C}_{26} \mathrm{H}_{21} \mathrm{~N}_{3} \mathrm{O}_{2} \\
(407.48)\end{array}$ & $\begin{array}{l}82.39 \\
82.59\end{array}$ & $\begin{array}{l}5.53 \\
5.45\end{array}$ & $\begin{array}{l}7.69 \\
7.44\end{array}$ & - & - \\
\hline $4 c$ & $3-\mathrm{CH}_{3}$ & - & 46 & $207-209$ & $\begin{array}{c}\mathrm{C}_{24} \mathrm{H}_{22} \mathrm{~N}_{4} \mathrm{O} \\
(382.47)\end{array}$ & $\begin{array}{l}85.17 \\
85.35\end{array}$ & $\begin{array}{l}6.55 \\
6.83\end{array}$ & $\begin{array}{l}8.28 \\
8.45\end{array}$ & - & - \\
\hline $4 d$ & $4-\mathrm{OCH}_{3}$ & - & 81 & 191-193 & $\begin{array}{c}\mathrm{C}_{24} \mathrm{H}_{22} \mathrm{~N}_{4} \mathrm{O}_{2} \\
(398.47)\end{array}$ & $\begin{array}{l}81.33 \\
81.22\end{array}$ & $\begin{array}{l}6.26 \\
6.52\end{array}$ & $\begin{array}{l}7.90 \\
7.71\end{array}$ & - & - \\
\hline $5 a$ & $\mathrm{H}$ & - & 64 & $234-236$ & $\begin{array}{c}\mathrm{C}_{23} \mathrm{H}_{18} \mathrm{~N}_{6} \\
(378.44)\end{array}$ & $\begin{array}{l}73.00 \\
73.26\end{array}$ & $\begin{array}{l}4.79 \\
4.87\end{array}$ & $\begin{array}{l}22.21 \\
22.73\end{array}$ & - & - \\
\hline $5 b$ & $2-\mathrm{CH}_{3}$ & - & 29 & 219-221 & $\begin{array}{c}\mathrm{C}_{24} \mathrm{H}_{20} \mathrm{~N}_{6} \\
(392.47)\end{array}$ & $\begin{array}{l}73.45 \\
73.54\end{array}$ & $\begin{array}{l}5.14 \\
4.88\end{array}$ & $\begin{array}{l}21.41 \\
21.78\end{array}$ & - & - \\
\hline $5 d$ & $4-\mathrm{OCH}_{3}$ & - & 72 & $228-230$ & $\begin{array}{c}\mathrm{C}_{24} \mathrm{H}_{20} \mathrm{~N}_{6} \mathrm{O} \\
(408.47)\end{array}$ & $\begin{array}{l}70.57 \\
70.41\end{array}$ & $\begin{array}{l}4.94 \\
4.67\end{array}$ & $\begin{array}{l}20.57 \\
20.31\end{array}$ & - & - \\
\hline $6 a$ & $\mathrm{H}$ & - & 86 & $262-264(14)$ & $\begin{array}{c}\mathrm{C}_{24} \mathrm{H}_{18} \mathrm{~N}_{4} \\
(362.44)\end{array}$ & - & - & - & - & - \\
\hline $6 b$ & $2-\mathrm{CH}_{3}$ & - & 39 & $218-220$ & $\begin{array}{c}\mathrm{C}_{25} \mathrm{H}_{20} \mathrm{~N}_{4} \\
(376.46)\end{array}$ & $\begin{array}{l}79.76 \\
79.42\end{array}$ & $\begin{array}{l}5.35 \\
5.17\end{array}$ & $\begin{array}{l}14.88 \\
15.13\end{array}$ & $\begin{array}{l}- \\
-\end{array}$ & - \\
\hline
\end{tabular}




\begin{tabular}{|c|c|c|c|c|c|c|c|c|c|c|}
\hline $6 c$ & $3-\mathrm{CH}_{3}$ & - & 58 & $235-237$ & $\begin{array}{c}\mathrm{C}_{25} \mathrm{H}_{20} \mathrm{~N}_{4} \\
(376.46)\end{array}$ & $\begin{array}{l}79.76 \\
79.53\end{array}$ & $\begin{array}{l}5.35 \\
5.71\end{array}$ & $\begin{array}{l}14.88 \\
14.52\end{array}$ & $\begin{array}{l}- \\
-\end{array}$ & - \\
\hline $6 d$ & $4-\mathrm{OCH}_{3}$ & - & 91 & $226-228$ & $\begin{array}{c}\mathrm{C}_{25} \mathrm{H}_{20} \mathrm{~N}_{4} \mathrm{O} \\
(392.46)\end{array}$ & $\begin{array}{l}76.51 \\
76.91\end{array}$ & $\begin{array}{l}5.14 \\
5.47\end{array}$ & $\begin{array}{l}14.28 \\
13.93\end{array}$ & $\begin{array}{l}- \\
-\end{array}$ & $\begin{array}{l}- \\
-\end{array}$ \\
\hline $7 a$ & $\mathrm{H}$ & - & 72 & $277-279$ & $\begin{array}{c}\mathrm{C}_{24} \mathrm{H}_{17} \mathrm{~N}_{3} \mathrm{O} \\
(363.42)\end{array}$ & $\begin{array}{l}79.32 \\
79.12\end{array}$ & $\begin{array}{l}4.72 \\
4.94\end{array}$ & $\begin{array}{l}11.56 \\
11.92\end{array}$ & $\begin{array}{l}- \\
-\end{array}$ & $\begin{array}{l}- \\
-\end{array}$ \\
\hline $7 b$ & $2-\mathrm{CH}_{3}$ & - & 44 & $253-255$ & $\begin{array}{c}\mathrm{C}_{25} \mathrm{H}_{19} \mathrm{~N}_{3} \mathrm{O} \\
(377.45)\end{array}$ & $\begin{array}{l}79.55 \\
79.66\end{array}$ & $\begin{array}{l}5.07 \\
5.39\end{array}$ & $\begin{array}{l}11.13 \\
10.75\end{array}$ & $\begin{array}{l}- \\
-\end{array}$ & $\begin{array}{l}- \\
-\end{array}$ \\
\hline $7 c$ & $3-\mathrm{CH}_{3}$ & - & 59 & $269-270$ & $\begin{array}{c}\mathrm{C}_{25} \mathrm{H}_{19} \mathrm{~N}_{3} \mathrm{O} \\
(377.45)\end{array}$ & $\begin{array}{l}79.55 \\
79.71\end{array}$ & $\begin{array}{l}5.07 \\
4.85\end{array}$ & $\begin{array}{l}11.13 \\
11.51\end{array}$ & $\begin{array}{l}- \\
-\end{array}$ & $\begin{array}{l}- \\
-\end{array}$ \\
\hline $7 \mathrm{~d}$ & $4-\mathrm{OCH}_{3}$ & - & 79 & $261-263$ & $\begin{array}{c}\mathrm{C}_{25} \mathrm{H}_{19} \mathrm{~N}_{3} \mathrm{O}_{2} \\
(393.45)\end{array}$ & $\begin{array}{l}76.32 \\
76.65\end{array}$ & $\begin{array}{l}4.87 \\
5.21\end{array}$ & $\begin{array}{l}10.68 \\
10.45\end{array}$ & - & $\begin{array}{l}- \\
-\end{array}$ \\
\hline $8 a$ & $\mathrm{H}$ & - & 81 & $230-232$ & $\begin{array}{c}\mathrm{C}_{24} \mathrm{H}_{18} \mathrm{~N}_{3} \mathrm{Cl} \\
(381.87)\end{array}$ & $\begin{array}{l}75.49 \\
75.43\end{array}$ & $\begin{array}{l}4.22 \\
4.59\end{array}$ & $\begin{array}{l}11.00 \\
11.23\end{array}$ & $\begin{array}{l}9.28 \\
8.96\end{array}$ & $\begin{array}{l}- \\
- \\
-\end{array}$ \\
\hline $8 b$ & $2-\mathrm{CH}_{3}$ & - & 55 & $217-219$ & $\begin{array}{c}\mathrm{C}_{25} \mathrm{H}_{18} \mathrm{~N}_{3} \mathrm{Cl} \\
(395.90)\end{array}$ & $\begin{array}{l}75.85 \\
75.88\end{array}$ & $\begin{array}{l}4.58 \\
4.67\end{array}$ & $\begin{array}{l}10.61 \\
10.66\end{array}$ & $\begin{array}{l}8.96 \\
8.71\end{array}$ & $\begin{array}{l}- \\
- \\
-\end{array}$ \\
\hline $8 c$ & $3-\mathrm{CH}_{3}$ & - & 67 & $221-223$ & $\begin{array}{c}\mathrm{C}_{25} \mathrm{H}_{18} \mathrm{~N}_{3} \mathrm{Cl} \\
(395.90)\end{array}$ & $\begin{array}{l}75.85 \\
75.71\end{array}$ & $\begin{array}{l}4.58 \\
4.92\end{array}$ & $\begin{array}{l}10.61 \\
10.79\end{array}$ & $\begin{array}{l}8.96 \\
9.12\end{array}$ & $\begin{array}{l}- \\
-\end{array}$ \\
\hline $8 d$ & $4-\mathrm{OCH}_{3}$ & - & 87 & 209-211 & $\begin{array}{c}\mathrm{C}_{25} \mathrm{H}_{18} \mathrm{~N}_{3} \mathrm{ClO} \\
(411.89)\end{array}$ & $\begin{array}{l}72.90 \\
73.24\end{array}$ & $\begin{array}{l}4.40 \\
4.21\end{array}$ & $\begin{array}{c}10.20 \\
9.85\end{array}$ & $\begin{array}{l}8.61 \\
8.48\end{array}$ & $\begin{array}{l}- \\
-\end{array}$ \\
\hline $9 b$ & $2-\mathrm{CH}_{3}$ & - & 76 & $227-229$ & $\begin{array}{c}\mathrm{C}_{25} \mathrm{H}_{19} \mathrm{~N}_{3} \mathrm{~S} \\
(393.51)\end{array}$ & $\begin{array}{l}76.31 \\
76.55\end{array}$ & $\begin{array}{l}4.87 \\
4.63\end{array}$ & $\begin{array}{l}10.68 \\
10.55\end{array}$ & $\begin{array}{l}- \\
-\end{array}$ & $\begin{array}{l}8.15 \\
8.36\end{array}$ \\
\hline $9 c$ & $3-\mathrm{CH}_{3}$ & - & 32 & $236-238$ & $\begin{array}{c}\mathrm{C}_{25} \mathrm{H}_{19} \mathrm{~N}_{3} \mathrm{~S} \\
(393.51)\end{array}$ & $\begin{array}{l}76.31 \\
76.10\end{array}$ & $\begin{array}{l}4.87 \\
5.12\end{array}$ & $\begin{array}{l}10.68 \\
10.47\end{array}$ & - & $\begin{array}{l}8.15 \\
7.88\end{array}$ \\
\hline $9 d$ & $4-\mathrm{OCH}_{3}$ & - & 46 & 205-207 & $\begin{array}{c}\mathrm{C}_{25} \mathrm{H}_{19} \mathrm{~N}_{3} \mathrm{SO} \\
(409.51)\end{array}$ & $\begin{array}{l}73.33 \\
73.41\end{array}$ & $\begin{array}{l}4.68 \\
4.59\end{array}$ & $\begin{array}{l}10.26 \\
10.39\end{array}$ & $\begin{array}{l}- \\
-\end{array}$ & $\begin{array}{l}7.83 \\
7.59\end{array}$ \\
\hline $10 a$ & $\mathrm{H}$ & $4-\mathrm{OCH}_{3} \mathrm{Ph}$ & 87 & $214-216$ & $\begin{array}{c}\mathrm{C}_{31} \mathrm{H}_{24} \mathrm{~N}_{4} \mathrm{O} \\
(468.56)\end{array}$ & $\begin{array}{l}79.47 \\
79.17\end{array}$ & $\begin{array}{l}5.16 \\
5.51\end{array}$ & $\begin{array}{l}11.96 \\
12.24\end{array}$ & $\begin{array}{l}- \\
-\end{array}$ & $\begin{array}{l}- \\
-\end{array}$ \\
\hline $10 \mathrm{~d}$ & $4-\mathrm{OCH}_{3}$ & $2-\mathrm{CH}_{3} \mathrm{Ph}$ & 64 & $225-227$ & $\begin{array}{c}\mathrm{C}_{32} \mathrm{H}_{26} \mathrm{~N}_{4} \mathrm{O} \\
(482.59)\end{array}$ & $\begin{array}{l}79.64 \\
79.24\end{array}$ & $\begin{array}{l}5.43 \\
5.62\end{array}$ & $\begin{array}{l}11.61 \\
11.40\end{array}$ & $\begin{array}{l}- \\
-\end{array}$ & $\begin{array}{l}- \\
-\end{array}$ \\
\hline
\end{tabular}

$4 \mathrm{~h}$, cooled, poured on ice-water to yield compounds 8a-d as precipitates, which were recrystallized from ethanol.

Synthesis of 7-aryl-5,6-diphenyl-7H-pyrrolo[2,3-d]pyrimidin-4(3H)-thione (9b-d)

A mixture of 4-chloropyrrolopyrimidines $\mathbf{8 b}, \mathbf{8 c}(3.95 \mathrm{~g}, 0.01 \mathrm{~mol})$ or $\mathbf{8 d}(4.11 \mathrm{~g}, 0.01$ $\mathrm{mol})$ and thiourea $(1.5 \mathrm{~g}, 0.02 \mathrm{~mol})$ was refluxed in dry ethanol $(30 \mathrm{~mL})$ for $4 \mathrm{~h}$. The solvent was removed under reduced pressure and the residue was recrystallized from methanol to give compounds $9 \mathbf{b}-\mathbf{d}$. 
Table II. Spectral data of the newly prepared compounds 1-10

\begin{tabular}{|c|c|c|c|}
\hline $\begin{array}{l}\text { Compd. } \\
\text { No. }\end{array}$ & $\begin{array}{c}\text { Mass } m / z \\
\text { (\% abundance) }\end{array}$ & $\operatorname{IR}\left(\mathrm{cm}^{-1}\right)$ & ${ }^{1} \mathrm{H}$ NMR (ppm) \\
\hline $1 \mathrm{a}$ & $335\left[\mathrm{M}^{+}\right](11.9 \%)$ & $\begin{array}{l}3566,3641\left(\mathrm{NH}_{2}\right) \\
2202(\mathrm{CN})\end{array}$ & $\begin{array}{l}5.04 \text { (br.s, } 2 \mathrm{H}, \mathrm{NH}_{2}, \mathrm{D}_{2} \mathrm{O} \text { exchangeable), } \\
7.04-7.67(\mathrm{~m}, 15 \mathrm{H}, \mathrm{Ar}-\mathrm{H})\end{array}$ \\
\hline $1 b$ & $\begin{array}{l}349\left[\mathrm{M}^{+}\right](100 \%) \\
350\left[\mathrm{M}^{+}+1\right](34.8 \%)\end{array}$ & $\begin{array}{l}3438,3315\left(\mathrm{NH}_{2}\right) \\
2209(\mathrm{CN})\end{array}$ & $\begin{array}{l}2.3\left(\mathrm{~s}, 3 \mathrm{H}, \mathrm{CH}_{3}\right), 6.07 \text { (br.s, } 2 \mathrm{H}, \mathrm{NH}_{2}, \mathrm{D}_{2} \mathrm{O} \\
\text { exchangeable), } 7.04-8.03(\mathrm{~m}, 14 \mathrm{H}, \mathrm{Ar}-\mathrm{H})\end{array}$ \\
\hline 1c & $\begin{array}{l}349\left[\mathrm{M}^{+}\right](100 \%) \\
350\left[\mathrm{M}^{+}+1\right](29.7 \%)\end{array}$ & $\begin{array}{l}3441,3315\left(\mathrm{NH}_{2}\right) \\
2210(\mathrm{CN})\end{array}$ & $\begin{array}{l}2.26\left(\mathrm{~s}, 3 \mathrm{H}, \mathrm{CH}_{3}\right), 5.09 \text { (br.s, } 2 \mathrm{H}, \mathrm{NH}_{2}, \mathrm{D}_{2} \mathrm{O} \\
\text { exchangeable), 6.5-7.9 (m, } 14 \mathrm{H}, \mathrm{Ar}-\mathrm{H})\end{array}$ \\
\hline $1 d$ & $365\left[\mathrm{M}^{+}\right](5.6 \%)$ & $\begin{array}{l}3526,3659\left(\mathrm{NH}_{2}\right) \\
2205(\mathrm{CN}) \\
1509(\mathrm{C}-\mathrm{O})\end{array}$ & $\begin{array}{l}3.79\left(\mathrm{~s}, 3 \mathrm{H}, \mathrm{OCH}_{3}\right), 5.1 \text { (br.s, } 2 \mathrm{H}, \mathrm{NH}_{2}, \mathrm{D}_{2} \mathrm{O} \\
\text { exchangeable), 6.6-7.8 (m, } 14 \mathrm{H}, \mathrm{Ar}-\mathrm{H})\end{array}$ \\
\hline $2 a$ & $353\left[\mathrm{M}^{+}\right](41 \%)$ & $\begin{array}{l}3350(\mathrm{~N}-\mathrm{H}) \\
1720(\mathrm{C}=\mathrm{O})\end{array}$ & $\begin{array}{l}5.0\left(\mathrm{~s}, 2 \mathrm{H}, \mathrm{NH}_{2}, \mathrm{D}_{2} \mathrm{O} \text { exchangeable }\right), 7.0-7.8 \\
(\mathrm{~m}, 15 \mathrm{H}, \mathrm{Ar}-\mathrm{H}), 8.55\left(\mathrm{~s}, 2 \mathrm{H}, \mathrm{CO}-\mathrm{NH}_{2}, \mathrm{D}_{2} \mathrm{O}\right. \\
\text { exchangeable })\end{array}$ \\
\hline $2 b$ & $367\left[\mathrm{M}^{+}\right](27 \%)$ & $\begin{array}{l}3347(\mathrm{~N}-\mathrm{H}) \\
1712(\mathrm{C}=\mathrm{O})\end{array}$ & $\begin{array}{l}2.26\left(\mathrm{~s}, 3 \mathrm{H}, \mathrm{Ph}-\mathrm{CH}_{3}\right), 5.09\left(\mathrm{~s}, 2 \mathrm{H}, \mathrm{NH}_{2}, \mathrm{D}_{2} \mathrm{O}\right. \\
\text { exchangeable }), 6.5-7.9(\mathrm{~m}, 14 \mathrm{H}, \text { Ar- } \mathrm{H}), \\
8.5\left(\mathrm{~s}, 2 \mathrm{H}, \mathrm{CO}-\mathrm{NH}_{2}, \mathrm{D}_{2} \mathrm{O} \text { exchangeable }\right)\end{array}$ \\
\hline $2 c$ & $367\left[\mathrm{M}^{+}\right](36 \%)$ & $\begin{array}{l}3410(\mathrm{~N}-\mathrm{H}) \\
1725(\mathrm{C}=\mathrm{O})\end{array}$ & $\begin{array}{l}2.3\left(\mathrm{~s}, 3 \mathrm{H}, \mathrm{Ph}-\mathrm{CH}_{3}\right), 6.0\left(\mathrm{~s}, 2 \mathrm{H}, \mathrm{NH}_{2}, \mathrm{D}_{2} \mathrm{O}\right. \\
\text { exchangeable), } 7.0-7.5(\mathrm{~m}, 14 \mathrm{H}, \text { Ar- } \mathrm{H}) \\
8.03\left(\mathrm{~s}, 2 \mathrm{H}, \mathrm{CO}-\mathrm{NH}_{2}, \mathrm{D}_{2} \mathrm{O} \text { exchangeable }\right)\end{array}$ \\
\hline $2 d$ & $383\left[\mathrm{M}^{+}\right](17 \%)$ & $\begin{array}{l}3392(\mathrm{~N}-\mathrm{H}) \\
1703(\mathrm{C}=\mathrm{O}) \\
1212(\mathrm{C}-\mathrm{O})\end{array}$ & $\begin{array}{l}2.25\left(\mathrm{~s}, 3 \mathrm{H}, \mathrm{Ph}-\mathrm{OCH}_{3}\right), 7.0-8.1(\mathrm{~m}, 14 \mathrm{H}, \mathrm{Ar}-\mathrm{H}), \\
4.8\left(\mathrm{~s}, 2 \mathrm{H}, \mathrm{NH}_{2}, \mathrm{D}_{2} \mathrm{O} \text { exchangeable }\right), 8.7(\mathrm{~s}, 2 \mathrm{H}, \\
\text { CO- } \mathrm{NH}_{2}, \mathrm{D}_{2} \mathrm{O} \text { exchangeable) }\end{array}$ \\
\hline $3 a$ & $377\left[\mathrm{M}^{+}\right](6.5 \%)$ & $\begin{array}{l}3410(\mathrm{NH}) \\
2225(\mathrm{CN}) \\
1712(\mathrm{C}=\mathrm{O})\end{array}$ & $\begin{array}{l}2.22\left(\mathrm{~s}, 3 \mathrm{H}, \mathrm{CH}_{3}-\mathrm{C}=\mathrm{O}\right), 6.9-8.0(\mathrm{~m}, 15 \mathrm{H}, \mathrm{Ar}-\mathrm{H}), \\
8.9\left(\mathrm{~s}, 1 \mathrm{H}, \mathrm{NH}, \mathrm{D}_{2} \mathrm{O} \text { exchangeable }\right)\end{array}$ \\
\hline $3 b$ & $391\left[\mathrm{M}^{+}\right](5.6 \%)$ & $\begin{array}{l}2223(\mathrm{CN}) \\
3423(\mathrm{NH}) \\
1717(\mathrm{C}=\mathrm{O})\end{array}$ & $\begin{array}{l}2.06\left(\mathrm{~s}, 3 \mathrm{H}, \mathrm{CH}_{3}-\mathrm{C}=\mathrm{O}\right), 2.27\left(\mathrm{~s}, 3 \mathrm{H}, \mathrm{Ph}-\mathrm{CH}_{3}\right) \\
6.9-7.9(\mathrm{~m}, 14 \mathrm{H}, \mathrm{Ar}-\mathrm{H}), 8.1\left(\mathrm{~s}, 1 \mathrm{H}, \mathrm{NH}, \mathrm{D}_{2} \mathrm{O}\right. \\
\text { exchangeable })\end{array}$ \\
\hline $3 c$ & $\begin{array}{l}391\left[\mathrm{M}^{+}\right](6.8 \%) \\
349\left[\mathrm{M}^{+}-\mathrm{Ac}\right](100 \%)\end{array}$ & $\begin{array}{l}3423(\mathrm{NH}) \\
2223(\mathrm{CN}) \\
1717(\mathrm{C}=\mathrm{O})\end{array}$ & $\begin{array}{l}2.12\left(\mathrm{~s}, 3 \mathrm{H}, \mathrm{CH}_{3}-\mathrm{C}=\mathrm{O}\right), 2.55\left(\mathrm{~s}, 3 \mathrm{H}, \mathrm{Ph}-\mathrm{CH}_{3}\right) \\
\text { 6.9-7.9 (m, } 14 \mathrm{H}, \mathrm{Ar}-\mathrm{H}), 8.3\left(\mathrm{~s}, 1 \mathrm{H}, \mathrm{NH}, \mathrm{D}_{2} \mathrm{O} \text { ex- }\right. \\
\text { changeable) }\end{array}$ \\
\hline $3 d$ & $\begin{array}{l}407\left[\mathrm{M}^{+}\right](33.9 \%) \\
365\left[\mathrm{M}^{+}-\mathrm{Ac}\right](100 \%)\end{array}$ & $\begin{array}{l}3373(\mathrm{NH}) \\
2137(\mathrm{CN}) \\
1510(\mathrm{C}-\mathrm{O}) \\
1709(\mathrm{C}=\mathrm{O}) \\
1709(\mathrm{C}=\mathrm{O})\end{array}$ & $\begin{array}{l}2.1\left(\mathrm{~s}, 3 \mathrm{H}, \mathrm{CH}_{3}-\mathrm{C}=\mathrm{O}\right), 3.75\left(\mathrm{~s}, 3 \mathrm{H}, \mathrm{CH}_{3}-\mathrm{O}\right) \\
6.8-7.9(\mathrm{~m}, 14 \mathrm{H}, \mathrm{Ar}-\mathrm{H}), 8.1\left(\mathrm{~s}, 1 \mathrm{H}, \mathrm{NH}, \mathrm{D}_{2} \mathrm{O}\right. \\
\text { exchangeable })\end{array}$ \\
\hline $4 c$ & $382\left[\mathrm{M}^{+}\right](43 \%)$ & $\begin{array}{l}3323 \text { broad } \\
(\mathrm{O}-\mathrm{H})\end{array}$ & $\begin{array}{l}2.34\left(\mathrm{~s}, 3 \mathrm{H}, \mathrm{CH}_{3}-\mathrm{Ph}\right), 4.2\left(\mathrm{~s}, 1 \mathrm{H}, \mathrm{OH}, \mathrm{D}_{2} \mathrm{O}\right. \\
\text { exchangeable), } 5.0 \text { (br.s, } 2 \mathrm{H}, \mathrm{NH}_{2}, \mathrm{D}_{2} \mathrm{O} \text { ex- } \\
\text { changeable), 6.5-7.4 (m, } 14 \mathrm{H}, \mathrm{Ar}-\mathrm{H}), 7.9(\mathrm{~s}, 1 \mathrm{H}, \\
\mathrm{NH}, \mathrm{D}_{2} \mathrm{O} \text { exchangeable), } 8.1\left(\mathrm{~s}, 1 \mathrm{H}, \mathrm{NH}, \mathrm{D}_{2} \mathrm{O}\right. \\
\text { exchangeable) }\end{array}$ \\
\hline $4 d$ & $398\left[\mathrm{M}^{+}\right](36.4 \%)$ & $\begin{array}{l}3423 \text { broad }(\mathrm{O}-\mathrm{H}) \\
1446(\mathrm{C}-\mathrm{O})\end{array}$ & $\begin{array}{l}3.8\left(\mathrm{~s}, 3 \mathrm{H}, \mathrm{OCH}_{3}\right), 5.2\left(\mathrm{~s}, 1 \mathrm{H}, \mathrm{OH}, \mathrm{D}_{2} \mathrm{O} \text { exchan- }\right. \\
\text { geable), } 5.9 \text { (br.s, } 2 \mathrm{H}, \mathrm{NH}_{2}, \mathrm{D}_{2} \mathrm{O} \text { exchangeable), } \\
\text { 6.7-8.1 (m, } 14 \mathrm{H}, \mathrm{Ar}-\mathrm{H}), 8.8\left(\mathrm{~s}, 1 \mathrm{H}, \mathrm{NH}, \mathrm{D}_{2} \mathrm{O} \text { ex- }\right. \\
\text { changeable), } 8.9\left(\mathrm{~s}, 1 \mathrm{H}, \mathrm{NH}, \mathrm{D}_{2} \mathrm{O} \text { exchangeable }\right)\end{array}$ \\
\hline
\end{tabular}




\begin{tabular}{|c|c|c|c|}
\hline $5 \mathbf{a}$ & $378\left[\mathrm{M}^{+}\right](76 \%)$ & $\begin{array}{l}3371(\mathrm{~N}-\mathrm{H}) \\
3435,3449\left(\mathrm{NH}_{2}\right)\end{array}$ & $\begin{array}{l}5.0\left(\mathrm{~s}, 2 \mathrm{H}, \mathrm{NH}_{2}, \mathrm{D}_{2} \mathrm{O} \text { exchangeable }\right) \\
7.0-7.6(\mathrm{~m}, 15 \mathrm{H}, \mathrm{Ar}-\mathrm{H}), 8.5(\mathrm{~s}, 1 \mathrm{H}, \mathrm{NH})\end{array}$ \\
\hline $5 b$ & $392\left[\mathrm{M}^{+}\right](28.9 \%)$ & $\begin{array}{l}3365(\mathrm{~N}-\mathrm{H}) \\
3451,3459\left(\mathrm{NH}_{2}\right)\end{array}$ & $\begin{array}{l}2.12\left(\mathrm{~s}, 3 \mathrm{H}, \mathrm{CH}_{3}\right), 5.2\left(\mathrm{~s}, 2 \mathrm{H}, \mathrm{NH}_{2}, \mathrm{D}_{2} \mathrm{O} \text { ex- }\right. \\
\text { changeable), } 7.0-7.65(\mathrm{~m}, 14 \mathrm{H}, \mathrm{Ar}-\mathrm{H}), 8.32(\mathrm{~s}, \\
1 \mathrm{H}, \mathrm{NH}, \mathrm{D}_{2} \mathrm{O} \text { exchangeable) }\end{array}$ \\
\hline $5 d$ & $408[\mathrm{M}+](60 \%)$ & $\begin{array}{l}3382(\mathrm{~N}-\mathrm{H}) \\
3526,3445\left(\mathrm{NH}_{2}\right) \\
1510(\mathrm{C}-\mathrm{O})\end{array}$ & $\begin{array}{l}3.76\left(\mathrm{~s}, 3 \mathrm{H}, \mathrm{OCH}_{3}\right), 5.2\left(\mathrm{~s}, 2 \mathrm{H}, \mathrm{NH}_{2}, \mathrm{D}_{2} \mathrm{O}\right. \\
\text { exchangeable }), 6.7-7.8(\mathrm{~m}, 14 \mathrm{H}, \mathrm{Ar}-\mathrm{H}) \\
8.36\left(\mathrm{~s}, 1 \mathrm{H}, \mathrm{NH}, \mathrm{D}_{2} \mathrm{O} \text { exchangeable }\right)\end{array}$ \\
\hline $6 \mathbf{a}$ & $361\left[\mathrm{M}^{+}\right](45.7 \%)$ & $3430,3330\left(\mathrm{NH}_{2}\right)$ & $\begin{array}{l}5.1 \text { (br.s, } 2 \mathrm{H}, \mathrm{NH}_{2}, \mathrm{D}_{2} \mathrm{O} \text { exchangeable) }, 7.1-7.6 \\
(\mathrm{~m}, 15 \mathrm{H}, \mathrm{Ar}-\mathrm{H}), 8.34(\mathrm{~s}, 1 \mathrm{H}, \mathrm{C}-2 \mathrm{H})\end{array}$ \\
\hline $6 b$ & $376\left[\mathrm{M}^{+}\right](30 \%)$ & $3447,3331\left(\mathrm{NH}_{2}\right)$ & $\begin{array}{l}2.54\left(\mathrm{~s}, 3 \mathrm{H}, \mathrm{CH}_{3}\right), 5.12 \text { (br.s, } 2 \mathrm{H}, \mathrm{NH}_{2}, \mathrm{D}_{2} \mathrm{O} \\
\text { exchangeable), } 7.0-7.9(\mathrm{~m}, 14 \mathrm{H}, \mathrm{Ar}-\mathrm{H}) \\
8.3(\mathrm{~s}, 1 \mathrm{H}, \mathrm{C}-2 \mathrm{H})\end{array}$ \\
\hline $6 c$ & $376\left[\mathrm{M}^{+}\right](11.5 \%)$ & $3415,3327\left(\mathrm{NH}_{2}\right)$ & $\begin{array}{l}2.31\left(\mathrm{~s}, 3 \mathrm{H}, \mathrm{CH}_{3}\right), 5.0 \text { (br.s, } 2 \mathrm{H}, \mathrm{NH}_{2}, \mathrm{D}_{2} \mathrm{O} \\
\text { exchangeable), } 7.0-7.5(\mathrm{~m}, 14 \mathrm{H}, \mathrm{Ar}-\mathrm{H}) \\
8.34\left(\mathrm{~s}, 1 \mathrm{H}, \mathrm{C}_{2}-\mathrm{H}\right)\end{array}$ \\
\hline $6 d$ & $392\left[\mathrm{M}^{+}\right](74.9 \%)$ & $3442,3346\left(\mathrm{NH}_{2}\right)$ & $\begin{array}{l}3.82\left(\mathrm{~s}, 3 \mathrm{H}, \mathrm{OCH}_{3}\right), 5.29 \text { (br.s, } 2 \mathrm{H}, \mathrm{NH}_{2}, \mathrm{D}_{2} \mathrm{O} \\
\text { exchangeable), 6.7-8.1 (m, } 14 \mathrm{H}, \mathrm{Ar}-\mathrm{H}) \\
8.8(\mathrm{~s}, 1 \mathrm{H}, \mathrm{C}-2 \mathrm{H})\end{array}$ \\
\hline $7 \mathbf{a}$ & $363\left[\mathrm{M}^{+}\right](46.3 \%)$ & $\begin{array}{l}3227(\mathrm{NH}) \\
1698(\mathrm{C}=\mathrm{O}) \\
1558(\mathrm{C}=\mathrm{N})\end{array}$ & $\begin{array}{l}\text { 6.9-7.9 (m, 15H, Ar-H), } 8.02(\mathrm{~s}, 1 \mathrm{H}, \mathrm{C}-2 \mathrm{H}) \\
8.2\left(\mathrm{~d}, 1 \mathrm{H}, \mathrm{NH}, \mathrm{D}_{2} \mathrm{O} \text { exchangeable }\right)\end{array}$ \\
\hline $7 \mathrm{~b}$ & $\begin{array}{l}377\left[\mathrm{M}^{+}\right](100 \%) \\
378\left[\mathrm{M}^{+}+1\right](22.7 \%)\end{array}$ & $\begin{array}{l}3328(\mathrm{NH}) \\
1686(\mathrm{C}=\mathrm{O}) \\
1572(\mathrm{C}=\mathrm{N})\end{array}$ & $\begin{array}{l}2.1\left(\mathrm{~s}, 3 \mathrm{H}, \mathrm{CH}_{3}\right), 7.2-8.0(\mathrm{~m}, 14 \mathrm{H}, \mathrm{Ar}-\mathrm{H}), 8.6(\mathrm{~d}, \\
\left.1 \mathrm{H}, \mathrm{NH}, \mathrm{D}_{2} \mathrm{O} \text { exchangeable }\right), 8.7(\mathrm{~s}, 1 \mathrm{H}, \mathrm{C}-2 \mathrm{H})\end{array}$ \\
\hline $7 \mathrm{c}$ & $377\left[\mathrm{M}^{+}\right](21 \%)$ & $\begin{array}{l}3362(\mathrm{NH}) \\
1679(\mathrm{C}=\mathrm{O}) \\
1598(\mathrm{C}=\mathrm{N})\end{array}$ & $\begin{array}{l}2.32\left(\mathrm{~s}, 3 \mathrm{H}, \mathrm{CH}_{3}\right), 6.9-7.67(\mathrm{~m}, 14 \mathrm{H}, \mathrm{Ar}-\mathrm{H}), 8.02 \\
\left(\mathrm{~d}, 1 \mathrm{H}, \mathrm{NH}, \mathrm{D}_{2} \mathrm{O} \text { exchangeable }\right), 8.3(\mathrm{~s}, 1 \mathrm{H}, \mathrm{C}-2 \mathrm{H})\end{array}$ \\
\hline $7 d$ & $393\left[\mathrm{M}^{+}\right](12.9 \%)$ & $\begin{array}{l}3130(\mathrm{NH}) \\
1682(\mathrm{C}=\mathrm{O}) \\
1587(\mathrm{C}=\mathrm{N}) \\
1510(\mathrm{C}-\mathrm{O})\end{array}$ & $\begin{array}{l}3.76\left(\mathrm{~s}, 3 \mathrm{H}, \mathrm{OCH}_{3}\right), 6.6-7.9(\mathrm{~m}, 14 \mathrm{H}, \mathrm{Ar}-\mathrm{H}), 8.1 \\
(\mathrm{~s}, 1 \mathrm{H}, \mathrm{C}-2 \mathrm{H}), 8.22\left(\mathrm{~s}, 1 \mathrm{H}, \mathrm{NH}, \mathrm{D}_{2} \mathrm{O} \text { exchange- }\right. \\
\text { able) }\end{array}$ \\
\hline $8 \mathbf{a}$ & $\begin{array}{l}381\left[\mathrm{M}^{+}\right](20 \%) \\
383[\mathrm{M}+2](8 \%)\end{array}$ & $\begin{array}{l}3076,2833(\mathrm{CH}) \\
1615(\mathrm{C}=\mathrm{C}) \\
1585(\mathrm{C}=\mathrm{N})\end{array}$ & $6.8-7.9(\mathrm{~m}, 15 \mathrm{H}, \mathrm{Ar}-\mathrm{H}), 8.7(\mathrm{~s}, 1 \mathrm{H}, \mathrm{C}-2 \mathrm{H})$ \\
\hline $8 b$ & $\begin{array}{l}395\left[\mathrm{M}^{+}\right](91 \%) \\
397[\mathrm{M}+2](28)\end{array}$ & $\begin{array}{l}3064,2846(\mathrm{CH}) \\
1619(\mathrm{C}=\mathrm{C}) \\
1571(\mathrm{C}=\mathrm{N})\end{array}$ & $\begin{array}{l}2.1\left(\mathrm{~s}, 3 \mathrm{H}, \mathrm{CH}_{3}\right), 7.2-8.0(\mathrm{~m}, 14 \mathrm{H}, \mathrm{Ar}-\mathrm{H}) \\
8.6(\mathrm{~s}, 1 \mathrm{H}, \mathrm{C}-2 \mathrm{H})\end{array}$ \\
\hline $8 c$ & $\begin{array}{l}395\left[\mathrm{M}^{+}\right](20 \%) \\
397[\mathrm{M}+2](5.4 \%)\end{array}$ & $\begin{array}{l}3056,2857(\mathrm{CH}) \\
1623(\mathrm{C}=\mathrm{C}) \\
1569(\mathrm{C}=\mathrm{N})\end{array}$ & $\begin{array}{l}2.3\left(\mathrm{~s}, 3 \mathrm{H}, \mathrm{CH}_{3}\right), 7.1-7.55(\mathrm{~m}, 14 \mathrm{H}, \mathrm{Ar}-\mathrm{H}) \\
7.9(\mathrm{~s}, 1 \mathrm{H}, \mathrm{C}-2 \mathrm{H})\end{array}$ \\
\hline $8 d$ & $\begin{array}{l}411\left[\mathrm{M}^{+}\right](40 \%) \\
413[\mathrm{M}+2](14.5 \%)\end{array}$ & $\begin{array}{l}3045,2926(\mathrm{CH}) \\
1629(\mathrm{C}=\mathrm{C}) \\
1605(\mathrm{C}=\mathrm{N}) \\
1226(\mathrm{C}-\mathrm{O})\end{array}$ & $\begin{array}{l}3.65\left(\mathrm{~s}, 3 \mathrm{H}, \mathrm{OCH}_{3}\right), 7.1-7.9(\mathrm{~m}, 14 \mathrm{H}, \mathrm{Ar}-\mathrm{H}) \\
8.6(\mathrm{~s}, 1 \mathrm{H}, \mathrm{C}-2 \mathrm{H})\end{array}$ \\
\hline $9 b$ & $393\left[\mathrm{M}^{+}\right](11.4 \%)$ & $\begin{array}{l}3362(\mathrm{~N}-\mathrm{H}) \\
1598(\mathrm{C}=\mathrm{N}) \\
3012,2980(\mathrm{C}-\mathrm{H})\end{array}$ & $\begin{array}{l}2.26\left(\mathrm{~s}, 3 \mathrm{H}, \mathrm{CH}_{3}\right), 6.9-7.7(\mathrm{~m}, 14 \mathrm{H}, \mathrm{Ar}-\mathrm{H}), 8.0(\mathrm{~s}, \\
1 \mathrm{H}, \mathrm{C}-2 \mathrm{H}), 8.1\left(\mathrm{~d}, 1 \mathrm{H}, \mathrm{NH}, \mathrm{D}_{2} \mathrm{O} \text { exchangeable) }\right.\end{array}$ \\
\hline
\end{tabular}




\begin{tabular}{|c|c|c|c|}
\hline 9c & $393\left[\mathrm{M}^{+}\right](18.7 \%)$ & $\begin{array}{l}1560(\mathrm{C}=\mathrm{N}) \\
3357(\mathrm{~N}-\mathrm{H}) \\
3023,2977(\mathrm{C}-\mathrm{H})\end{array}$ & $\begin{array}{l}2.05\left(\mathrm{~s}, 3 \mathrm{H}, \mathrm{CH}_{3}\right), 6.8-7.7(\mathrm{~m}, 14 \mathrm{H}, \mathrm{Ar}-\mathrm{H}), 8.4(\mathrm{~s}, \\
1 \mathrm{H}, \mathrm{C}-2 \mathrm{H}), 8.8\left(\mathrm{~d}, 1 \mathrm{H}, \mathrm{NH}, \mathrm{D}_{2} \mathrm{O} \text { exchangeable }\right)\end{array}$ \\
\hline $9 d$ & $409\left[\mathrm{M}^{+}\right](6.5 \%)$ & $\begin{array}{l}3368(\mathrm{~N}-\mathrm{H}) \\
1586(\mathrm{C}=\mathrm{N}) \\
3025,2983(\mathrm{C}-\mathrm{H})\end{array}$ & $\begin{array}{l}3.7\left(\mathrm{~s}, 3 \mathrm{H}, \mathrm{OCH}_{3}\right), 6.8-7.8(\mathrm{~m}, 14 \mathrm{H}, \mathrm{Ar}-\mathrm{H}), 8.0 \\
(\mathrm{~s}, 1 \mathrm{H}, \mathrm{C}-2 \mathrm{H}), 9.5\left(\mathrm{~d}, 1 \mathrm{H}, \mathrm{NH}, \mathrm{D}_{2} \mathrm{O} \text { exchange- }\right. \\
\text { able) }\end{array}$ \\
\hline 10a & $468\left[\mathrm{M}^{+}\right](46.7 \%)$ & $\begin{array}{l}1554(\mathrm{C}=\mathrm{N}) \\
3347(\mathrm{~N}-\mathrm{H}) \\
1221(\mathrm{C}-\mathrm{O})\end{array}$ & $\begin{array}{l}3.77\left(\mathrm{~s}, 3 \mathrm{H}, \mathrm{OCH}_{3}\right), 6.5-7.5(\mathrm{~m}, 19 \mathrm{H}, \mathrm{Ar}-\mathrm{H}), 8.4 \\
(\mathrm{~s}, 1 \mathrm{H}, \mathrm{C}-2 \mathrm{H}), 8.6\left(\mathrm{~d}, 1 \mathrm{H}, \mathrm{NH}, \mathrm{D}_{2} \mathrm{O} \text { exchangeable }\right)\end{array}$ \\
\hline $10 \mathrm{~d}$ & $482\left[\mathrm{M}^{+}\right](21 \%)$ & $\begin{array}{l}1567(\mathrm{C}=\mathrm{N}) \\
3352(\mathrm{~N}-\mathrm{H}) \\
1217(\mathrm{C}-\mathrm{O})\end{array}$ & $\begin{array}{l}2.5\left(\mathrm{~s}, 3 \mathrm{H}, \mathrm{CH}_{3}\right), 3.8\left(\mathrm{~s}, 3 \mathrm{H}, \mathrm{OCH}_{3}\right), 6.9-7.6(\mathrm{~m}, \\
18 \mathrm{H}, \mathrm{Ar}-\mathrm{H}), 8.0(\mathrm{~s}, 1 \mathrm{H}, \mathrm{C}-2 \mathrm{H}), 8.9(\mathrm{~d}, 1 \mathrm{H}, \mathrm{NH}, \\
\left.\mathrm{D}_{2} \mathrm{O} \text { exchangeable }\right)\end{array}$ \\
\hline
\end{tabular}

\section{Synthesis of $\mathrm{N}-(7-a r y l-5,6-d i p h e n y l-7 \mathrm{H}-p y r r o l o[2,3-\mathrm{d}] p y$ rimidine)-N-aryl amines} $(10 a, d)$

A mixture of the appropriate 4-chloropyrrolopyrimidine, $8 \mathbf{a}(3.81 \mathrm{~g}, 0.01 \mathrm{~mol})$ or $\mathbf{8 d}$ $(4.11 \mathrm{~g}, 0.01 \mathrm{~mol})$, aryl amine $[p$-anisidine $(1.23 \mathrm{~g}, 0.01 \mathrm{~mol})$ or $m$-toluidine $(1.07 \mathrm{~g}, 0.01)$ and a few drops of triethylamine was refluxed in absolute ethanol for $8 \mathrm{~h}$. The solvent was removed under reduced pressure and the residues were recrystallized from methanol to give $10 a, d$.

\section{Antimicrobial evaluation}

Antimicrobial activity of the synthesized compounds in comparison with that of some control drugs [amoxicilin (Bioanalyse, Turkey) and fluconazol (Sigma-Aldrich, USA)] was evaluated against Gram positive (Staphylococcus aureus, ATCC 29213, Bacillus subtilis, ATCC 6633), Gram-negative (Escherichia coli, ATCC 25922, Pseudomonas aeruginosa, ATCC 278533) bacteria and fungus (Candida albicans, ATCC 10231). All microorganisms used were obtained from the culture collection of the Department of Microbiology and Immunology, Faculty of Pharmacy, Helwan University, Cairo, Egypt. The microorganisms were passaged at least twice to ensure purity and viability. The lowest concentration of the compound that completely inhibited macroscopic growth was determined and minimum inhibitory concentrations (MIC) was reported. MIC for each tested compound was determined on Mueller-Hinton agar (MHA) by the microdilution technique according to NCCLS guidelines 1997 (15).

All bacterial isolates were subcultured in MHA plates and incubated overnight at $37^{\circ} \mathrm{C}$ and Candida isolates were subcultured in Sabouraud-dextrose agar (SDA) plates at $35^{\circ} \mathrm{C}$ for $24-48 \mathrm{~h}$.

Solution of the newly synthesized compounds and standard drugs were prepared at concentrations of $1024,512,256,128,64,32$ and $16 \mu \mathrm{g} \mathrm{mL}-1$ using serial two-folds dilutions in DMSO. Each concentration was mixed with sterile nutrient agar (Sigma-Aldrich, USA) in a sterile plate and bacteria inoculum was added to each well of the microdilution tray. The trays were incubated at $37^{\circ} \mathrm{C}$ in a humid chamber and MIC endpoints were read after $24 \mathrm{~h}$ of incubation. 
DMSO (80 \%, Sigma-Aldrich), pure microorganisms, and pure media were used as controls.

Testing the synergistic activity. - To test the synergistic activity of active tested compounds, a mixture of two compounds (1:1 ratio) was prepared in the concentrations of $1024,512,256,128,64,32$ and $16 \mu \mathrm{g} \mathrm{mL}^{-1}$, and was tested for by the same method as mentioned before for their antimicrobial activity against the same bacterial and fungal strains (Table III).

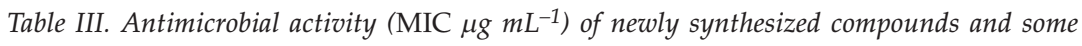
mixtures of the synthesized compounds

\begin{tabular}{|c|c|c|c|c|c|}
\hline Compd. No. & B. subtilis & S. aureus & E. coli & P. aeruginosa & C. albicans \\
\hline $1 b$ & - & - & 32 & - & 32 \\
\hline $1 c$ & 64 & 256 & 128 & - & 128 \\
\hline 1d & 256 & - & - & - & 256 \\
\hline $2 b$ & - & - & 64 & - & 32 \\
\hline $3 a$ & - & - & - & - & - \\
\hline $3 b$ & - & - & 128 & - & 32 \\
\hline $3 c$ & 64 & - & 128 & - & 64 \\
\hline $3 d$ & - & - & 128 & - & 64 \\
\hline $4 c$ & 128 & - & - & - & - \\
\hline $5 d$ & - & - & 64 & - & - \\
\hline $6 a$ & - & - & - & 64 & - \\
\hline $6 b$ & 32 & 256 & - & - & 128 \\
\hline $6 c$ & 128 & 128 & - & - & 128 \\
\hline $7 a$ & 128 & - & 256 & - & 128 \\
\hline $7 \mathrm{c}$ & 256 & - & - & - & 256 \\
\hline $7 d$ & 64 & - & - & - & 32 \\
\hline $9 b$ & - & - & - & - & 32 \\
\hline $10 a$ & - & - & - & - & 32 \\
\hline $1 c+3 c$ & 32 & - & 64 & - & 64 \\
\hline $6 c+7 a$ & 64 & - & 512 & - & 64 \\
\hline $3 b+3 d$ & - & - & 128 & - & 64 \\
\hline $1 b+7 d$ & 128 & - & 64 & - & 32 \\
\hline $1 c+6 b$ & 64 & - & 256 & - & 128 \\
\hline $9 b+10 a$ & - & - & - & - & 32 \\
\hline Amoxicillin & 64 & 16 & 256 & 64 & - \\
\hline Fluconazol & - & - & - & - & 512 \\
\hline
\end{tabular}


RESULTS AND DISCUSSION

\section{Chemistry}

Condensation of benzoin with primary aromatic amines in refluxing toluene resulted in the formation of $\alpha$-aminoketone intermediates, which were condensed, without isolation, with malononitrile to yield the various 2-amino-4,5-diphenylpyrrole-3-carbonitriles (1a-d). Pyrroles 1a-d reacted with concentrated sulphuric acid to yield 2-amino-3-carbamoyl-4,5-diphenylpyrroles (2a-d). Acetylated pyrrole-3-carbonitriles (3a-d) were obtained by the reaction of $\mathbf{1 a - d}$ with acetic anhydride.

Preparation of 3-(2-amino-4,5-diphenylpyrrole)amidoximes (4c,d) was accomplished by the reaction of $\mathbf{1} \mathbf{c}, \mathbf{d}$ with hydroxylaminehydrochloride in absolute ethanol using sodium carbonate. On the other hand, synthesis of 2-amino-4,5-diphenyl-3-tetrazolo-pyrroles $(\mathbf{5} \mathbf{a}, \mathbf{b}, \mathbf{d})$ was achieved by the reaction of $\mathbf{1} \mathbf{a}, \mathbf{b}, \mathbf{d}$ with sodium azide in dimethyl formamide using ammonium chloride (Scheme 1).

On the other hand, pyrrolopyrimidines $6 a-d$ and $7 \mathrm{a}-\mathrm{d}$ were obtained by the reaction of 1a-d with formamide and formic acid, respectively; 4-chloro-pyrrolo-pyrimidines $\mathbf{8 a}-\mathbf{d}$ were prepared by the reaction of $\mathbf{7 a - d}$ with phosphorus oxychloride as previously described for the preparation of analogous compounds $(14,16-19)$.

The pyrrolo[2,3- $d]$ pyrimidin-4(3H)-thiones $\mathbf{9 b}$-d were obtained by the reaction of $\mathbf{8 b}-\mathbf{d}$ with thiourea in absolute ethanol. Finally, $N$-(pyrrolo[2,3- $d$ ]pyrimidines)- $N$-aryl amines $10 \mathrm{a}, \mathrm{d}$ were obtained from compounds $8 \mathrm{a}, \mathrm{d}$ by the previously described method for the preparation of analogous compounds (16) (Scheme 2).

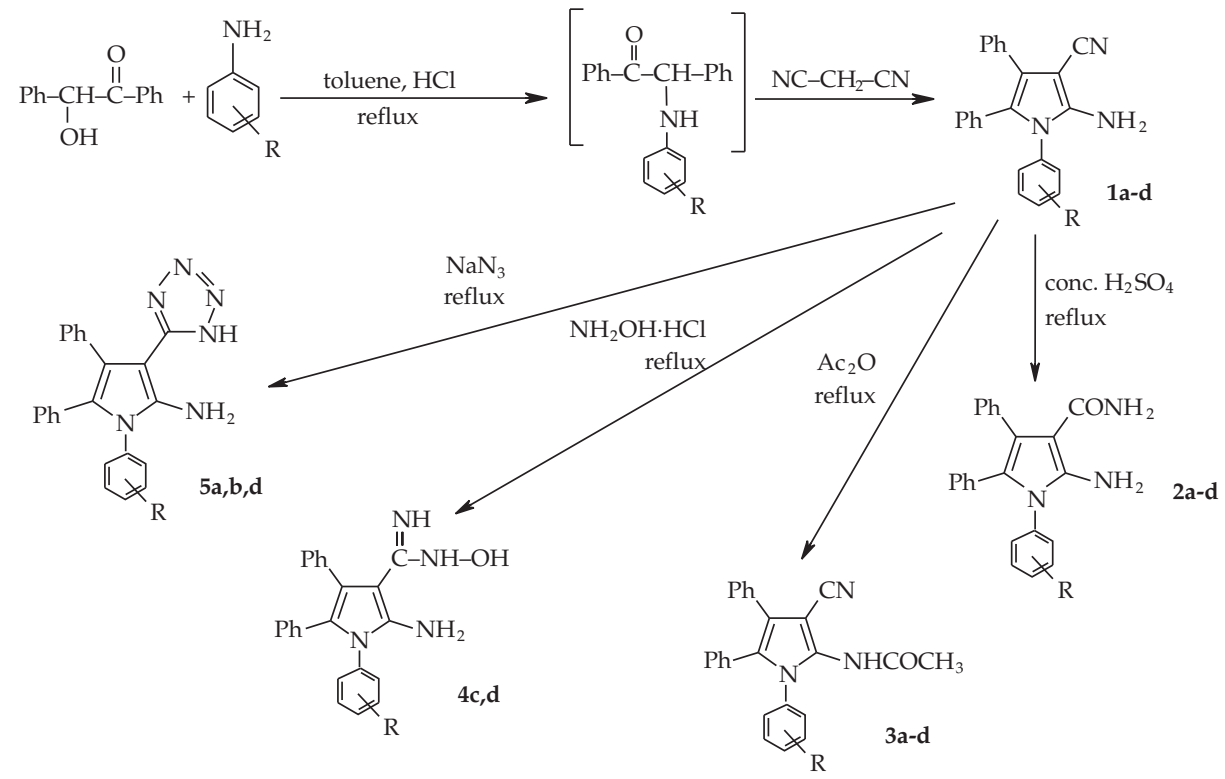

Scheme 1 


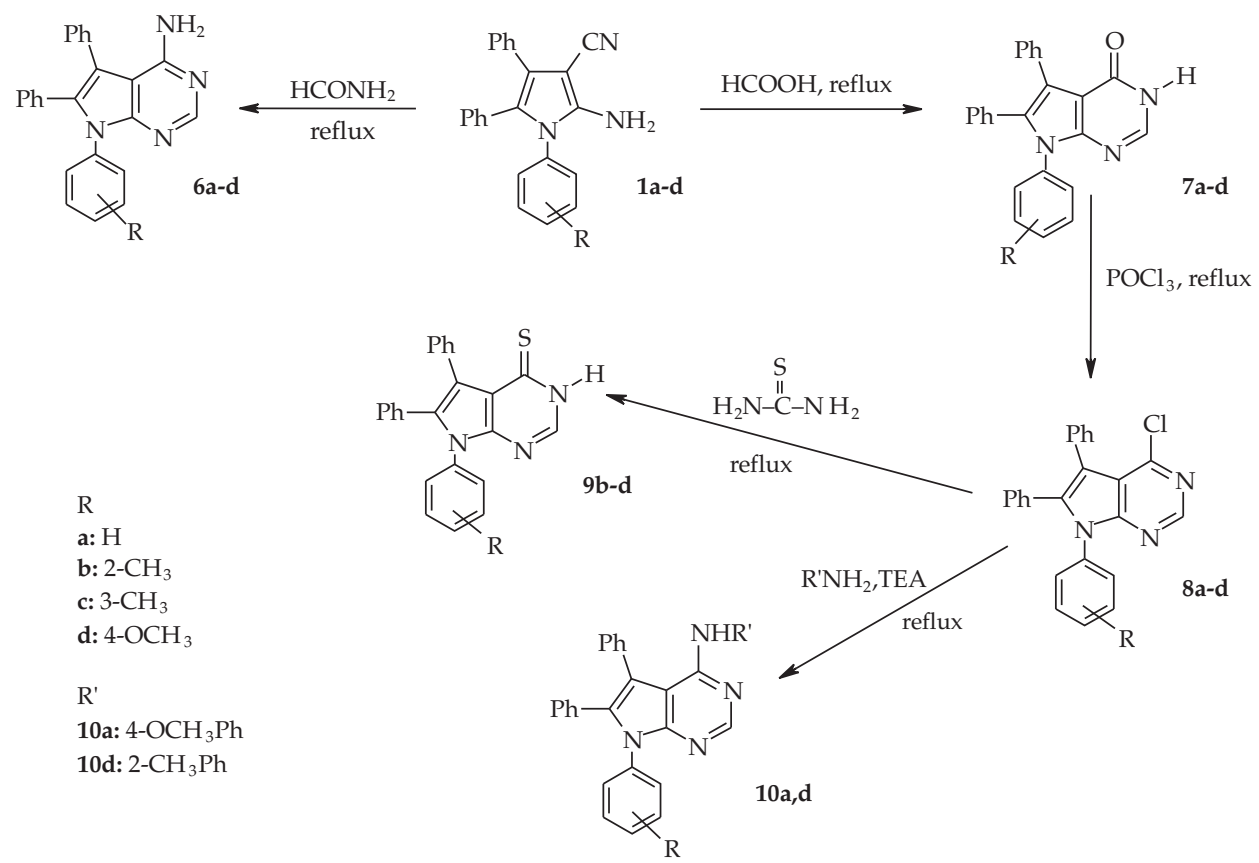

Scheme 2

Structures of the synthesized compounds were confirmed by microanalytical and spectral data (Tables I and II).

\section{Antimicrobial activity}

The antimicrobial activity of some newly synthesized compounds revealed that compounds $1 \mathrm{c}, 3 \mathrm{c}$, and $7 \mathrm{~d}$ were similar in their activity to amoxicillin against Bacillus subtilis (MIC $64 \mu \mathrm{g} \mathrm{mL}^{-1}$ ), while compound $6 \mathrm{~b}$ was two times more active than amoxicillin against the same organism (Table III). On the other hand, compound $6 \mathrm{c}$ showed lower activity than amoxicillin against Staphylococcus aureus (MIC 128 vs. $16 \mu \mathrm{g} \mathrm{mL}^{-1}$ ).

Furthermore, determination of antibacterial activity against Escherichia coli indicated that compounds $\mathbf{2} \mathbf{b}$ and $\mathbf{5 d}$ were four times as active as amoxicillin (MIC $64 \mu \mathrm{g} \mathrm{mL}^{-1}$ ), while compound $\mathbf{1 b}$ was even more active against Escherichia coli (MIC $\left.32 \mu \mathrm{g} \mathrm{mL}^{-1}\right)$. Compound 6a exhibited significant activity against Pseudomonas aeruginosa, aquivalent to that of amoxicillin (20).

The results indicated that compounds $\mathbf{1 b}, \mathbf{2 b}, \mathbf{3 b}, \mathbf{7 d}, \mathbf{9 b}$ and $\mathbf{1 0 a}$ were more active against Candida albicans (MIC $32 \mu \mathrm{g} \mathrm{mL}^{-1}$ ) when compared with fluconazol (MIC $512 \mu \mathrm{g}$ $\left.\mathrm{mL}^{-1}\right)$. Compounds $3 \mathrm{c}$ and $3 \mathrm{~d}$ were also highly active (MIC $64 \mu \mathrm{g} \mathrm{mL}^{-1}$ ) and compounds $\mathbf{1 c}, \mathbf{6 b}, \mathbf{6 c}$, and $\mathbf{7 a}$ were four times as active as fluconazol (MIC $128 \mu \mathrm{g} \mathrm{mL}^{-1}$ ) whereas 
compounds 1d and 7c were two times more active than fluconazol (MIC $\left.256 \mu \mathrm{g} \mathrm{mL}^{-1}\right)$. The other compounds did not show significant antibacterial/antimycotic activity.

From the above results, we can see that 2-amino-1,4,5-triphenyl-pyrrole-3-carbonitrile (1a) is totally inactive against Gram-positive, Gram-negative bacteria and fungi. On replacing phenyl group at position 1 with 2-methylphenyl in compound $\mathbf{1} \mathbf{b}$, it acquired high antimicrobial activity against Escherichia coli and Candida albicans, and on replacing the phenyl group with 3-methylphenyl (in 1c), it acquired mild antimicrobial activity against Escherichia coli, Bacillus subtilis and Candida albicans. Replacing this phenyl group by 4-methoxyphenyl and cyano group in position 3 with tetrazole ring (in $\mathbf{5 d}$ ) led to high antibacterial activity against Escherichia coli.

On the other hand, on converting pyrrole 1a to 4-amino-pyrrolo[2,3- $d]$ pyrimidine (6a), it acquired significant activity against Pseudomonas aeruginosa pyrrolo[2,3- $d]$ pyrimidine-4-one (7a) showed mild activity against Bacillus subtilis and Candida albicans. It was found that pyrrolo[2,3- $d]$ pyrimidine-4-thione $(9 \mathbf{b})$ acquired high antifungal activity against Candida albicans. When the slightly active pyrrole derivative $1 \mathbf{d}$ was converted to pyrrolopyrimidine-4-one (7d), it acquired significant antimicrobial activity against Bacillus subtilis and Candida albicans. On replacing the ketonic oxygen atom of $7 \mathbf{a}$ by arylamine in 10a, it lost its activity against Bacillus subtilis but was still active against Candida albicans.

The synergistic test revealed that the mixture of compounds $1 \mathrm{c}$ and $3 \mathrm{c}$ acquired higher activity against Bacillus subtilis (MIC $32 \mu \mathrm{g} \mathrm{mL}-1)$, Escherichia coli (MIC $64 \mu \mathrm{g} \mathrm{mL}^{-1}$ ) and Candida albicans (MIC $64 \mu \mathrm{g} \mathrm{mL}^{-1}$ ) than that of individual compounds (Table III).

The mixture of compounds $\mathbf{6 c}$ and $7 \mathbf{a}$ acquired higher activity against Bacillus subtilis (MIC $64 \mu \mathrm{g} \mathrm{mL}^{-1}$ ) and Candida albicans (MIC $64 \mu \mathrm{g} \mathrm{mL}^{-1}$ ) than that of individual compounds.

The mixtures of compounds $3 b$ and $3 d, 1 b$ and $7 d, 1 c$ and $6 b$, and $9 b$ and $10 a$ showed similar activities as those of individual compounds (Table III).

\section{CONCLUSIONS}

Evaluation of the novel compounds established that some of the synthesized compounds, namely, [2-amino-1-(2-methylphenyl)-4,5-diphenyl- $1 H$-pyrrole-3-carbonitriles (1b), 2-amino-3-carbamoyl-1-(3-methylphenyl)-4,5-diphenyl-1H-pyrroles (2b), $\mathrm{N}$-(3-cyano-1-(2-methylphenyl)-4,5-diphenyl-1H-pyrrol-2-yl)-acetamide (3b), $\mathrm{N}$-(3-cyano-1-(3-methylphenyl)-4,5-diphenyl-1H-pyrrol-2-yl)-acetamide (3c), 2-amino-1-(4-methoxyphenyl)-4,5-diphenyl-3-tetrazolo-1H-pyrrole (5d), 7-(4-methoxyphenyl)-5,6-diphenyl-7H-pyrrolo[2,3-d]pyrimidin-4(3H)-one $(7 \mathrm{~d})]$ showed improved antimicrobial activity compared to amoxicillin, while the other compounds, 7-(3-methylphenyl)-5,6-diphenyl-7H-pyrrolo [2,3-d]pyrimidin-4(3H)-thione $(9 \mathbf{b})$ and $\mathrm{N}$-(7-(2-methylphenyl)-5,6-diphenyl-7H-pyrrolo $[2,3-d]$ pyrimidine)- $N$-aryl amine (10a), were highly active antimycotic agents compared to fluconazole.

Acknowledgements. - The autors express their sincere thaks to all the professors of the Microanalytical Center, Faculty of Science, Cairo University, Cairo, Egypt, for providing the elemental, IR, mass and NMR analyses. 


\section{REFERENCES}

1. S. Petruso, S. Bonanno, S. Caronna, M. Ciofalo, B. Maggio and D. Schillaci, A new synthesis of bioactive analogs of monodeoxypyoluteorin, J. Heterocyclic Chem. 31 (1994) 941-945.

2. M. V. Raimondi, S. Cascioferro, D. Schillaci and S. Petruso, Synthesis and antimicrobial activity of new bromine-rich pyrrole derivatives related to monodeoxypyoluteorin, Eur. J. Med. Chem. 41 (2006) 1439-1445; DOI 10.1016/j.ejmech.2006.0.009.

3. D. Schillaci, S. Petruso and V. Sciortino, 3,4,5,3,5-Pentabromo-2-(2-hydroxybenzoyl)pyrrole: a potential lead compound as anti-Gram-positive and anti-biofilm agent, Int. J. Antimicrob. Ag. 25 (2005) 338-340; DOI: 10.1016/j.ijantimicag.2004.11.014.

4. M. Biava, R. Fioravanti, G. C. Porretta, D. Deidda, C. Maullu and R. Pompei, New pyrrole derivatives as antimycobacterial agents analogs of BM 212, Bioorg. Med. Chem. Lett. 9 (1999) 2983-2988; DOI: 10.1016/s0960-894x(99)00510-7.

5. M. Biava, G. C. Porretta, D. Deidda, R. Pompei, A. Tafi and F. Manetti, Importance of the thiomorpholine introduction in new pyrrole derivatives as antimycobacterial agents analogues of BM 212, Bioorg. Med. Chem. 11 (2003) 515-520; DOI: 10.1016/s0968-0896(02)00455-8.

6. G. H. Jana, S. Jain, S. K. Arora and N. Sinha, Synthesis of some diguanidino 1-methyl-2,5-diaryl-1H-pyrroles as antifungal agents, Bioorg. Med. Chem. Lett. 15 (2005) 3592-3595; DOI: 10.1016/ j.bmcl.2005.05.080.

7. Y. Misumo, M. J. Ikehara, K. A. Watanabe, S. Suzuki and T. Itoh, Synthetic studies of potential antimetabolites. IX. The anomeric configuration of tubercidin, J. Org. Chem. 28 (1969) 3329-3331.

8. Q. Dang and J. E. Gomez-Galeno, An efficient synthesis of pyrrolo[2,3-d]pyrimidines via inverse electron demand Diels-Alder reactions of 2-amino-4-cyanopyrroles with 1,3,5-triazines, J. Org. Chem. 67 (2002) 8703-8705; DOI: 10.1021/jo026309d.

9. K. V. Rao, Structure of sangivamycin, J. Med. Chem. 11 (1968) 939-941; DOI: 10.1021/jm00311a005.

10. A. Gangjee, X. Lin and S. F. Queener, Design, synthesis, and biological evaluation of 2,4-diamino-5-methyl-6-substituted-pyrrolo[2,3-d]pyrimidines as dihydrofolate reductase inhibitors, J. Med. Chem. 47 (2004) 3689-3692; DOI: 10.1021/jm0306327.

11. P. M. Traxler, P. Furet, H. Mett, E. Buchdunger, T. Meyer and N. Lydon, 4-(Phenylamino)pyrrolopyrimidines: Potent and selective, ATP site directed inhibitors of the EGF-receptor protein tyrosine kinase, J. Med. Chem. 39 (1996) 2285-2290; DOI: 10.1021/jm960118j.

12. M. S. Mohamed, A. E. Rashad, M. E. A. Zaki and S. S. Fatahala, Synthesis and antimicrobial screening of some fused heterocyclic pyrroles, Acta Pharm. 55 (2005) 237-249.

13. H. J. Roth und K. Eger, Synthese von 2-Amino-3-cyano-pyrrolen, Arch. Pharm. 18 (1974) 179-185.

14. H. J. Roth und K. Eger, Synthese von pyrrolo[2,3-d]pyrimidinen, Arch. Pharm. 18 (1974) $252-258$.

15. A. C. Gales, H. S. Sader and R. N. Jones, Urinary tract infection trends in Latin American hospitals: report from the SENTRY antimicrobial surveillance program, Diagn. Mic. Infec. Dis. 44 (1997-2000) 289-294.

16. A. Jorgensen, K. A. M. El-Bayouki and E. B. Pedersen, Phosphorus pentoxide in organic synthesis. XX: Synthesis of N-aryl-7H-pyrrolo[2,3-d]pyrimidin-4-amines, J. Heterocyclic Chem. 22 (1985) 859-863.

17. C. G. Dave and M. C. Shukla, Diethyl ethoxymethylenemalonate in triheterocycles: a new synthesis of pyrido[3,2-e]pyrimido[1,2-c]pyrimidines, J. Heterocyclic Chem. 34 (1997) 1805-1808.

18. C. G. Dave and R. D. Shah, Synthesis of 7H-tetrazolo[1,5-c]pyrrolo[3,2-e]pyrimidines and their reductive ring cleavage to 4-aminopyrrolo[2,3-d]pyrimidines, J. Heterocyclic Chem. 35 (1998) 1295-1300. 
19. C. G. Dave, Synthesis and reactions of fluoroaryl substituted 2-amino-3-cyanopyrroles and pyrrolo[2,3-d]pyrimidines, J. Heterocyclic Chem. 36 (1999) 729-733.

20. G. F. Gad, R. A. El-Domany and H. M. Ashour, Antimicrobial susceptibility profile of Pseudomonas aeruginosa isolates in Egypt, J. Urol. 180 (2008) 176-181; DOI: 10.1016/j.juro.2008.03.081.

\author{
$S A \check{Z} E T A K$
}

\title{
Sinteza derivata pirola kao antimikrobnih tvari
}

MOSAAD SAYED MOHAMED, RAMDAN AHMED EL-DOMANY i RANIA HELMY ABD EL-HAMEED

U radu je opisana sinteza novih derivata pirola i pirolo[2,3-d]pirimidina s poboljšanim antimikrobnim djelovanjem. Reakcijom benzoina, primarnih aromatskih amina i malononitrila sintetizirana je serija novih 2-aminopirol-3-karbonitrila (1a-d), iz kojih su potom pripravljeni derivati pirola (2a-d do $5 \mathbf{a}-\mathbf{d})$ i pirolo[2,3-d]pirimidina (6a-d do $\mathbf{1 0 a}, \mathbf{d})$. Sintetiziranim spojevima ispitano je in vitro antimikrobno djelovanje na različite Gram-pozitivne, Gram-negativne bakterije i gljivice. Neki od sintetiziranih spojeva [2-amino-1-(2-metilfenil)-4,5-difenil-1H-pirol-3-karbonitril (1b), 2-amino-3-karbamoil-1-(3-metilfenil)-4,5-difenil-1H-pirol (2b), $N$-(3-cijano-1-(2-metilfenil)-4,5-difenil-1H-pirol-2-il)-acetamid (3b), $N$-(3-cijano-1-(3-metilfenil)-4,5-difenil-1H-pirol-2-il)-acetamid (3c), 2-amino1-(4-metoksifenil)-4,5-difenil-3-tetrazolo-1H-pirol (5d), 7-(4-metoksifenil)-5,6-difenil-7Hpirolo[2,3-d]pirimidin-4(3H)-on (7d), 7(3-metilfenil)-5,6-difenil-7H-pirolo[2,3-d]pirimidin$4(3 H)$-tion (9b) i N-(7-(2-metilfenil)-5,6-difenil-7H-pirolo[2,3-d]pirimidin)-N-aril amin (10a)] imaju snažno antimikrobno djelovanje.

Ključne riječi: pirol, pirolopirimidin, antimikrobno djelovanje

Organic Chemistry Department, Faculty of Pharmacy, Helwan University, Cairo, Egypt

Microbiology and Immunology Department, Faculty of Pharmacy Helwan University, Cairo, Egypt 